\title{
Trends in worldwide nanotechnology patent applications: 1991 to 2008
}

\author{
Yan Dang $\cdot$ Yulei Zhang $\cdot$ Li Fan • \\ Hsinchun Chen · Mihail C. Roco
}

Received: 7 December 2009/ Accepted: 10 December 2009/Published online: 29 December 2009

(C) Springer Science+Business Media B.V. 2009

\begin{abstract}
Nanotechnology patent applications published during 1991-2008 have been examined using the "title-abstract" keyword search on esp@cenet "worldwide" database. The longitudinal evolution of the number of patent applications, their topics, and their respective patent families have been evaluated for 15 national patent offices covering $98 \%$ of the total global activity. The patent offices of the United States (USA), People's Republic of China (PRC), Japan, and South Korea have published the largest number of nanotechnology patent applications, and experienced significant but different growth rates after 2000. In most repositories, the largest numbers of nanotechnology patent applications originated from their own countries/regions, indicating a significant "home
\end{abstract}

Y. Dang $(\bowtie) \cdot$ Y. Zhang $\cdot$ L. Fan $\cdot$ H. Chen

Department of Management Information Systems, Eller

College of Management, The University of Arizona,

Tucson, AZ 85721, USA

e-mail: ydang@email.arizona.edu

Y. Zhang

e-mail: ylzhang@email.arizona.edu

L. Fan

e-mail: fanli@email.arizona.edu

H. Chen

e-mail: hchen@eller.arizona.edu

M. C. Roco

National Science Foundation, 4201 Wilson Blvd.,

Arlington, VA 22230, USA

e-mail: mroco@nsf.gov advantage." The top applicant institutions are from different sectors in different countries (e.g., from industry in the US and Canada patent offices, and from academe or government agencies at the PRC office). As compared to 2000, the year before the establishment of the US National Nanotechnology Initiative (NNI), numerous new invention topics appeared in 2008, in all 15 patent repositories. This is more pronounced in the USA and PRC. Patent families have increased among the 15 patent offices, particularly after 2005. Overlapping patent applications increased from none in 1991 to about $4 \%$ in 2000 and to about $27 \%$ in 2008 . The largest share of equivalent nanotechnology patent applications $(1,258)$ between two repositories was identified between the US and Japan patent offices.

Keywords Nanotechnology - Research and development·esp@cenet "worldwide" database . Number of patent applications - Longitudinal evolution · Patent topics · Patent family analysis

\section{Introduction}

Nanotechnology developments continue to be produced at exponential rates for a wide and diverse range of applications. Because of nanotechnology's pivotal role in future scientific and economic development, in order to remain competitive, more than 60 countries have adopted national projects or programs 
to prompt research in nanotechnology since 2000 (Roco 2005). Patent analysis is an indicator for assessing development of different research communities and technology fields (Narin 1998; Oppenheim 2000). Our evaluation framework integrates bibliographic, citation (Garfield 1955), contents (Tolle and Chen 2000), network (Albert and Barabasi 2002), and statistical analyses. The framework has been applied to nanotechnology patent publications in the US Patent and Trademark Office (USPTO), the European Patent Office (EPO), and the Japan Patent Office (JPO) in Huang et al. (2003) (for USPTO), Huang et al. (2006) (for USPTO), and Li et al. (2007) (for USPTO, EPO, and JPO).

This study has considered all the public repositories and is focused on data from the 15 countries'/regions' repositories that are most active in the nanotechnology domain; these repositories cover $98 \%$ of the patent applications worldwide. The respective applications were searched on titles and abstracts ("title-abstract" search) using a group of keywords provided by domain experts (Huang et al. 2003). This article presents the longitudinal evolution of the number of patent applications after their origin, topics, and corresponding patent families since 1991.

\section{Analysis method of patent applications}

Data collection and preprocessing

Nanotechnology publications from different countries'/regions' patent offices (repositories) were extracted from the esp@cenet "worldwide" database into our database by using keyword "title-abstract" searching.

A patent office is a governmental or intergovernmental organization which controls the issue of patents. Different countries have their own patent offices, such as the USPTO, the JPO, the Canadian Intellectual Property Office (CIPO), and the South Korean Intellectual Property Office (KIPO). In addition to national (country level) patent offices, there are several regional (country group level) patent offices as well, such as the EPO and the World Intellectual Property Organization (WIPO). The EPO grants European patents for the 27 member states of the European Patent Convention. The WIPO is a specialized agency of the United Nations with 184 member states in 2008. It grants patents for all of its member states. Many countries publish patent applications and/ or grant patent rights for public information (Chemical Abstracts Service 2008).

A reliable international database covering patent information from multiple patent offices is the esp@ cenet "worldwide" database, which is maintained by the EPO together with the member states of the European Patent Organization. Esp@cenet includes three databases:

- "EPO" database

- "WIPO" database

- "worldwide" database

The esp@cenet "worldwide" database contains the patent applications examined and published by 85 individual countries'/regions' patent offices, including the USPTO, EPO, and JPO. The esp@cenet "worldwide" database holds more than 60 million patents (Espacenet Website, "Coverage of the worldwide database") (Espacenet Website, "Coverage of the worldwide database") (EPO 2008). English translations for all other languages are provided for the bibliographic information, and selected content information (such as abstract, claim, and description) are also provided. Owing to the limitation of the search functions of esp@cenet, we collected the patent applications by searching the nanotechnology keywords only in each patent application's title and abstract ("title-abstract" search).

The esp@cenet "worldwide" database previously has been used to examine patents in biology (Oldham and Cutter 2006), hydrogen and fuel cells (Seymour et al. 2007), and globalization of knowledge (Andersen et al. 2006).

\section{Patent parsing}

Two sets of patent information were parsed into our database from the collected patent applications:

- Nanotechnology patent applications published in different countries'/regions' patent offices (repositories)

- Patent family information of these patent applications.

Table 1 shows the data field limitation of our patent application collection. Most of the data fields are available in the esp@cenet "worldwide" database. 
Table 1 Data field limitation of the esp@cenet "worldwide" database

\begin{tabular}{ll}
\hline Data field & Available \\
\hline Publication number & Yes \\
Publication date & Yes \\
Inventor name & Yes \\
Applicant institution name & Yes \\
Applicant country & $*$ \\
International patent classification code (IPC) & Yes \\
European patent classification code (EPC) & $*$ \\
Citation information & No \\
Priority number(s) & Yes \\
Title & Yes \\
Abstract & Yes \\
Claim & $*$ \\
Description & $*$ \\
\hline
\end{tabular}

* The patent application data from some countries/regions' patent offices is incomplete

However, it does not contain the citation information for patent applications published in patent offices other than EPO and WIPO (Espacenet Website, "What is a cited document?"). For some regional or country patent offices, the bibliographic data (such as the application country, European patent classification code (EPC), claim, or description) are incomplete. All the selected repositories in our study are part of EPO and WIPO.

A patent application for an invention is originally filed in one country; however, it can be filed later in other countries as well. The original, first application filing generally is considered to be the priority application (Hingley and Park 2003). In esp@cenet, such related applications or "members of corresponding documents" or "equivalents" and have exactly the same priority (Espacenet Website, "Also published as documents").

A patent family is a group of patents that are all related to each other. We use the esp@cenet patent "simple family" definition as comprising all the documents having exactly the same priority or combination of priorities (Espacenet Website, "Patent families"). The International Patent Documentation Center (INPADOC) defines as "expanded family" all the documents sharing directly or indirectly (e.g., via a third document) at least one priority (Espacenet Website, "Patent families").
Data analysis

Three types of analyses were conducted using the data collected from the previous components:

- Longitudinal evolution of the number of patent publications per year and per applicant (i.e., the institution to which a patent is assigned to countries, applicant institutions, and technology fields)

- Topic analysis, where we have created content maps to identify the most important and emerging research topics in nanotechnology domain in different time intervals for each patent office (repository).

- Patent family analysis across different patent offices (repositories) including ranking those with the largest numbers of equivalent patent applications.

\section{Results}

Data description

We collected the nanotechnology patent applications published from 1991 to 2008 from the esp@cenet "worldwide" database. We focused attention on the leading 15 country/regional patent offices that cover more than $98 \%$ of the whole collection; each has more than 100 patent applications.

Longitudinal evolution of patent applications

\section{Global increase of nanotechnology patents}

The evolution of the total number of nanotechnology patent applications in the 15 repositories per year from 1991 to 2008 is shown in Fig 1. This figure also shows the number of non-overlapping nanotechnology patent applications by considering one patent application per family. The annual rate of increase for all the patent publications is more pronounced between 2000 and 2008 (34.5\%). This rate is higher than that of Science Citation Index's article publication rate of $20-25 \%$ for the same period when we use the same keyword "title-abstract" search approach as for patent applications.

The percentage of nanotechnology patent application as compared to the total number of patent applications in all the technical areas is illustrated in Fig. 2. 
Fig. 1 Longitudinal evolution of the total number of nanotechnology patent applications in the 15 repositories per year ("title abstract," 1991-2008)

Fig. 2 Longitudinal evolution of the percentage of patent publications on nanotechnology versus all topics, in the repositories of leading 15 countries/regions and USA from 1991 to 2008 using keyword "titleabstract" search
Total number of nanotechnology applications per year

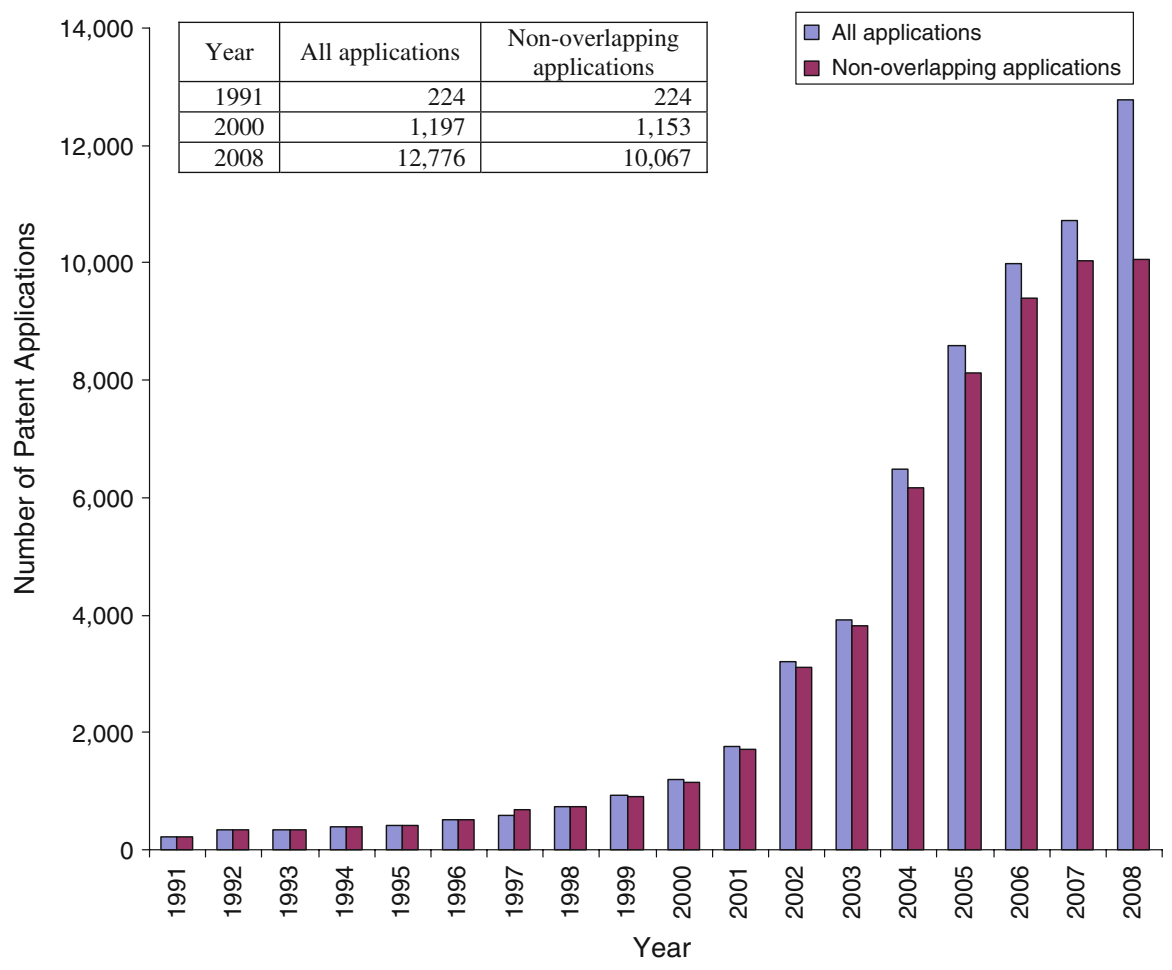

The percentage of nanotechnology patent application as compared to the total number of patent applications in all technical areas

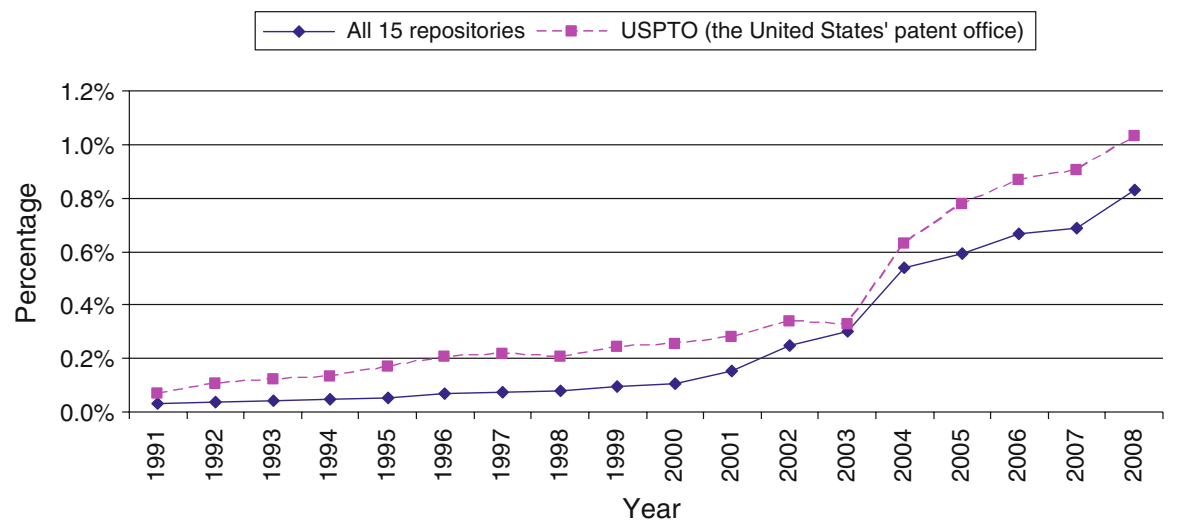

The nanotechnology patent application percentages for the USPTO reported in the above figure are consistent with the data trends reported in previous studies (Huang et al. 2006; Hu et al. 2007) where the granted patents were searched by "title-claims" from 1991 to 2004. In that study, the percentage of granted patents reached $1.09 \%$ in 2004 versus $0.63 \%$ for patent applications in Fig. 2. Our previous studies also showed that the nanotechnology-granted patent percentages for "full-text" search was $4.85 \%$ in 2004 for the USPTO.

\section{Number of patent applications per repository}

Table 2 lists the numbers of nanotechnology patent applications published by each of the 15 countries/ 
Table 2 Nanotechnology patent applications published in the top 15 countries/regions' patent offices in the interval 1991 to 2008 using keyword "title-abstract" search

\begin{tabular}{llrrr}
\hline Rank & $\begin{array}{l}\text { Patent office } \\
\text { (repository) }\end{array}$ & $\begin{array}{l}\text { No. of nanotechnology } \\
\text { patent applications (1991-2008) }\end{array}$ & 2000 & 2008 \\
\hline 1 & USA & 19,665 & 405 & 3,729 \\
2 & PRC & 18,438 & 105 & 5,030 \\
3 & Japan & 10,763 & 328 & 1,744 \\
4 & South Korea & 5,963 & 74 & 1,249 \\
5 & Canada & 1,539 & 41 & 255 \\
6 & Taiwan & 1,363 & 28 & 3 \\
7 & Germany & 1,312 & 62 & 70 \\
8 & Australia & 1,296 & 76 & 136 \\
9 & Russian Federation & 859 & 45 & 162 \\
10 & Mexico & 471 & 0 & 88 \\
11 & UK & 412 & 14 & 68 \\
12 & France & 390 & 8 & 38 \\
13 & Brazil & 315 & 0 & 103 \\
14 & Ukraine & 243 & 0 & 83 \\
15 & New Zealand & 140 & 11 & 18 \\
\hline
\end{tabular}

regions' patent offices from 1991 to 2008. The USPTO examined and published the largest number of nanotechnology patent applications, followed by the patent offices of the PRC and Japan.

The total number of nanotechnology patent applications published from 1991 to 2008 by authors from the US and PRC are estimated each at over 17,000. Over $20 \%$ of the US patent applications and $4 \%$ of the PRC's are in foreign repositories.

Figures 3 and 4 show the evolution of the numbers of nanotechnology patent applications published in different countries'/regions' patent offices by year. Since the patent offices of the US, PRC, Japan, and South Korea had many more nanotechnology patent applications, we present their evolution trends in Fig. 3. The evolution trends of the other 11 countries'/regions' patent offices are shown in Fig. 4.

The patent offices of the US, PRC, Japan, and South Korea have significantly more nanotechnology patent applications than other patent offices, and all experienced larger increases especially after 2003. The PRC's repository surpassed the USA' repository after 2006. As shown in Fig. 4, the other 11 patent offices have experienced mostly increases but also decreased in recent years. The patent offices of the Russian Federation, Brazil, and the United Kingdom (UK) reached their peaks in 2008 with 162, 103, and 68 nanotechnology patent applications, respectively. The Ukraine's patent office peaked in 2007 with 87

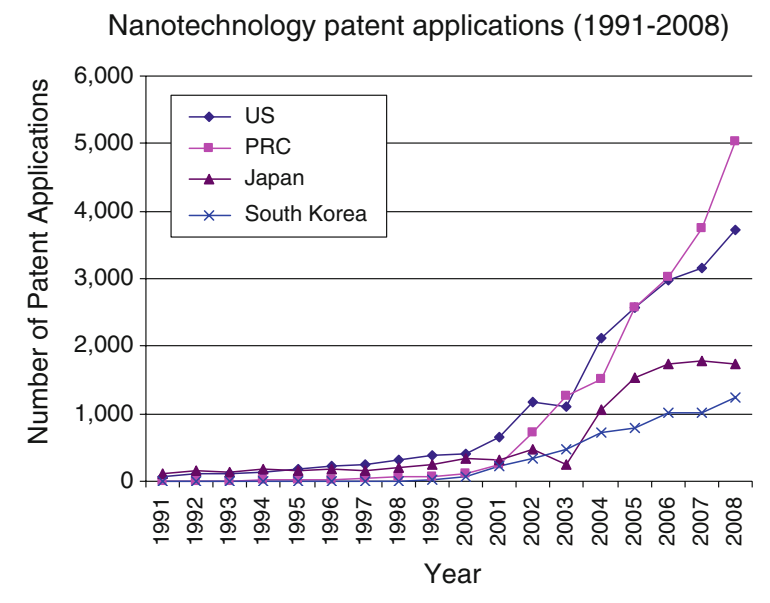

Fig. 3 The numbers of nanotechnology patent applications from all countries in the patent offices of the US, PRC, Japan, and South Korea using "title-abstract" search, from 1991 to 2008

nanotechnology patent applications, and the patent offices of Germany and New Zealand reached their peaks in 2006 with 164 and 21 nanotechnology patent applications, respectively. Canada's and Mexico's patent offices reached their peaks in 2005 with 274 and 94 nanotechnology patent applications, respectively. Australia's and France's patent offices peaked in 2003 with 343 and 57 nanotechnology patent applications, respectively. Taiwan's patent office had more than 200 nanotechnology patent applications per year from 
Fig. 4 The numbers of nanotechnology patent applications from all the countries in the remaining 11 patent offices using "title-abstract" search from 1991 to 2008
Nanotechnology patent applications (1991-2008)

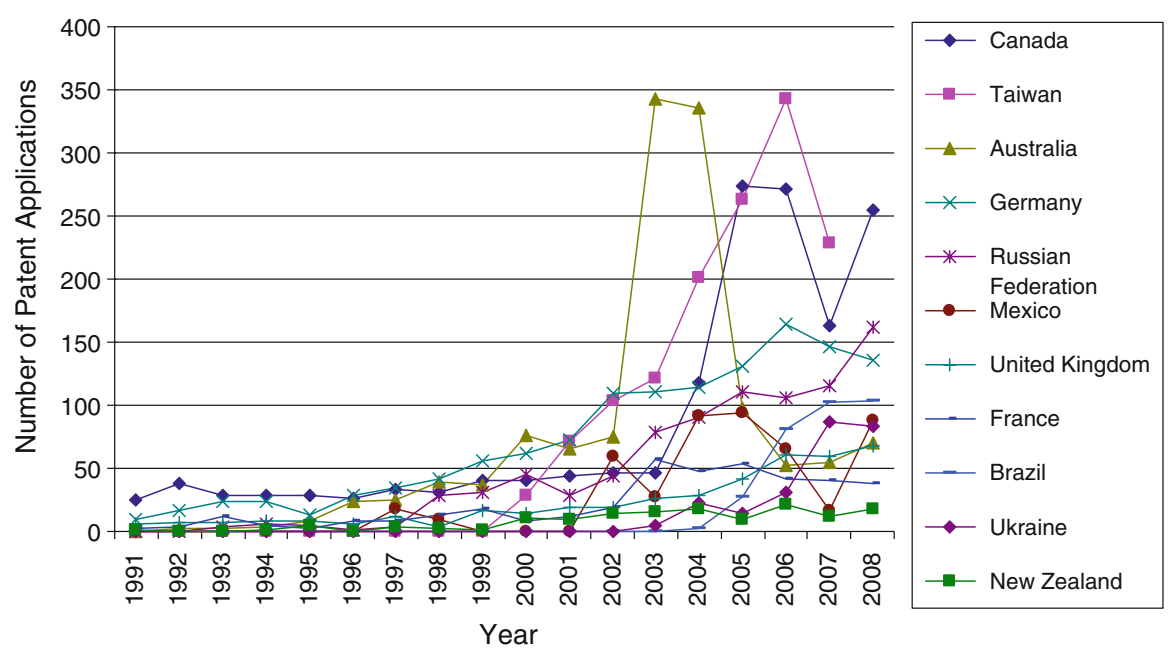

2004 to 2007 with 2006 as the peak (343 applications); however, the number dropped dramatically in 2008 to only three nanotechnology patent applications probably due to a delay in collecting the 2008 Taiwan patent data by the esp@cenet "worldwide" database. In all following analyses, we used 2007 data for Taiwan's patent office instead of 2008.

Most patent offices generally publish the country of origin of the patent publications, with the exceptions of Japan, Australia, and New Zealand. Table 3 lists the top five countries where patent applications were filled from 1991 to 2008. For several of the other patent offices, a small portion of their patent applications may have incomplete applicant country information. In these cases, we manually verified the information. For each application having the applicant same as the inventor(s), we then used the country of the first inventor as its applicant country. As a comparison, we also list the numbers of nanotechnology patent applications published in 2000 (the year before the establishment of the US National Nanotechnology Initiative; Roco et al. 2000) and 2008 (the most recent year with data available for the whole year).

The USA was the most active internationally with the largest numbers of nanotechnology patent applicants published in other patent offices. It ranked first in three out of the 12 patent offices, including its own patent office, Canada's, and Mexico's patent offices; it ranked second in the patent offices of six other countries; and third in the remaining three patent offices. Japan, Germany, South Korea, and France are the most active internationally after the USA.
These results on country ranking generally are consistent with those reported in the previous study on granted patents at USPTO using "title-claims" search (Li et al. 2007), in which study the top five applicant countries of USPTO nanotechnology-granted patents published from 1976 to 2004 were the US (3,450 patents), Japan (517 patents), Germany (204 patents), France (156 patents), and South Korea (131 patents), with Taiwan being the seventh. In this study, the top five applicant countries identified were the US, Japan, South Korea, Germany, and Taiwan. However, the numbers of nanotechnology patent documents reported in this study are different from those reported by Li et al. (2007) due to three reasons. First, instead of using the granted patents as used by Li et al. (2007), we used the published patent applications as the data source in this study, because the esp@cenet "worldwide" database does not differentiate granted patents from published patent applications. Second, in this study involving 15 repositories we could not use the more complete "title/abstract/claims" used in previous study only for the USPTO. Third, our results are based on the data published from 1991 to 2008 while the numbers reported by Li et al. (2007) are based on the data published from 1976 to 2004. Many patent offices have published a large number of nanotechnology patents in recent years.

Table 3 shows that all the patent offices except those of Canada and Mexico had the largest numbers of nanotechnology patent applications published by applicants from their own countries. This indicates a "home advantage" effect. As defined in previous 
Table 3 Top five applicant countries in 12 countries/regions' patent offices based on the number of nanotechnology patent applications from 1991 to 2008

\begin{tabular}{|c|c|c|c|c|c|c|}
\hline No. & $\begin{array}{l}\text { Patent office/repository } \\
\text { (no. of applications } \\
\text { from all countries) }\end{array}$ & Rank & $\begin{array}{l}\text { Applicant } \\
\text { country }\end{array}$ & $\begin{array}{l}\text { Number of nanotechnology } \\
\text { patent applications } \\
(1991-2008)\end{array}$ & 2000 & 2008 \\
\hline \multirow[t]{5}{*}{1} & \multirow[t]{5}{*}{ USA $(19,665)$} & 1 & USA & 12,606 & 285 & 2,288 \\
\hline & & 2 & Japan & 1,866 & 42 & 308 \\
\hline & & 3 & South Korea & 1,272 & 6 & 343 \\
\hline & & 4 & Germany & 1,048 & 23 & 168 \\
\hline & & 5 & Taiwan & 839 & 7 & 175 \\
\hline \multirow[t]{5}{*}{2} & \multirow[t]{5}{*}{ PRC $(18,438)$} & 1 & PRC & 16,348 & 85 & 4,409 \\
\hline & & 2 & USA & 805 & 3 & 260 \\
\hline & & 3 & South Korea & 327 & 5 & 80 \\
\hline & & 4 & Japan & 301 & 2 & 64 \\
\hline & & 5 & Germany & 145 & 3 & 43 \\
\hline \multirow[t]{5}{*}{3} & \multirow[t]{5}{*}{ South Korea $(5,963)$} & 1 & South Korea & 4,087 & 41 & 967 \\
\hline & & 2 & USA & 461 & 15 & 151 \\
\hline & & 3 & PRC & 145 & 1 & 53 \\
\hline & & 4 & Japan & 138 & 4 & 39 \\
\hline & & 5 & Germany & 119 & 0 & 42 \\
\hline \multirow[t]{5}{*}{4} & \multirow[t]{5}{*}{ Canada $(1,539)$} & 1 & USA & 825 & 18 & 156 \\
\hline & & 2 & Canada & 192 & 4 & 28 \\
\hline & & 3 & Germany & 124 & 7 & 18 \\
\hline & & 4 & France & 57 & 6 & 7 \\
\hline & & 5 & Japan & 53 & 2 & 5 \\
\hline \multirow[t]{5}{*}{5} & \multirow[t]{5}{*}{ Taiwan $(1,363)^{\mathrm{a}}$} & 1 & Taiwan & 906 & 3 & 165 \\
\hline & & 2 & USA & 224 & 17 & 26 \\
\hline & & 3 & Japan & 113 & 3 & 17 \\
\hline & & 4 & Germany & 35 & 3 & 6 \\
\hline & & 5 & South Korea & 32 & 2 & 10 \\
\hline \multirow[t]{5}{*}{6} & \multirow[t]{5}{*}{ Germany $(1,312)$} & 1 & Germany & 1,182 & 56 & 124 \\
\hline & & 2 & Taiwan & 21 & 1 & 1 \\
\hline & & 3 & USA & 20 & 1 & 3 \\
\hline & & 4 & South Korea & 16 & 2 & 2 \\
\hline & & 5 & Japan & 15 & 0 & 1 \\
\hline \multirow[t]{5}{*}{7} & \multirow[t]{5}{*}{ Russian Federation (859) } & 1 & Russian Federation & 711 & 41 & 147 \\
\hline & & 2 & USA & 37 & 1 & 3 \\
\hline & & 3 & Japan & 17 & 0 & 3 \\
\hline & & 3 & Germany & 17 & 1 & 1 \\
\hline & & 5 & France & 16 & 0 & 2 \\
\hline \multirow[t]{5}{*}{8} & \multirow[t]{5}{*}{ Mexico (471) } & 1 & USA & 277 & 0 & 53 \\
\hline & & 2 & Germany & 30 & 0 & 5 \\
\hline & & 3 & Mexico & 28 & 0 & 4 \\
\hline & & 4 & France & 26 & 0 & 3 \\
\hline & & 5 & Switzerland & 20 & 0 & 2 \\
\hline
\end{tabular}


Table 3 continued

\begin{tabular}{|c|c|c|c|c|c|c|}
\hline No. & $\begin{array}{l}\text { Patent office/repository } \\
\text { (no. of applications } \\
\text { from all countries) }\end{array}$ & Rank & $\begin{array}{l}\text { Applicant } \\
\text { country }\end{array}$ & $\begin{array}{l}\text { Number of nanotechnology } \\
\text { patent applications } \\
(1991-2008)\end{array}$ & 2000 & 2008 \\
\hline \multirow[t]{5}{*}{9} & \multirow[t]{5}{*}{ UK (412) } & 1 & UK & 162 & 5 & 27 \\
\hline & & 2 & USA & 109 & 8 & 15 \\
\hline & & 3 & Germany & 25 & 0 & 7 \\
\hline & & 4 & Japan & 20 & 0 & 3 \\
\hline & & 5 & South Korea & 19 & 1 & 5 \\
\hline \multirow[t]{5}{*}{10} & \multirow[t]{5}{*}{ France (390) } & 1 & France & 358 & 6 & 37 \\
\hline & & 2 & Belgium & 6 & 0 & 2 \\
\hline & & 2 & Japan & 6 & 0 & 0 \\
\hline & & 4 & Taiwan & 5 & 0 & 0 \\
\hline & & 4 & USA & 5 & 2 & 0 \\
\hline \multirow[t]{5}{*}{11} & \multirow[t]{5}{*}{ Brazil (315) } & 1 & Brazil & 116 & 0 & 42 \\
\hline & & 2 & USA & 99 & 0 & 32 \\
\hline & & 3 & Germany & 25 & 0 & 8 \\
\hline & & 4 & Switzerland & 21 & 0 & 4 \\
\hline & & 5 & France & 15 & 0 & 5 \\
\hline \multirow[t]{5}{*}{12} & \multirow[t]{5}{*}{ Ukraine (243) } & 1 & Ukraine & 221 & 0 & 78 \\
\hline & & 2 & Switzerland & 4 & 0 & 3 \\
\hline & & 3 & Japan & 2 & 0 & 0 \\
\hline & & 4 & Switzerland & 1 & 0 & 0 \\
\hline & & 4 & USA & 1 & 0 & 0 \\
\hline
\end{tabular}

a As noted previously, data from 2007 was used for Taiwan's patent office, rather than 2008

studies, the "home advantage" effect is the tendency of domestic applicants to file more patents with their home country patent office than foreign applicants (European Commission 1997; Ganguli 1998; Criscuolo 2005).

By comparing the numbers of nanotechnology patent applications published in 2000 and 2008, the tremendous increase in nanotechnology patent applications from each top applicant country can be easily perceived. Especially notable are the increases recorded by Mexico, Brazil, and Ukraine.

\section{Top applicant institutions}

Table 4 lists the leading five applicant institutions per repository that includes large companies, universities, and research centers. In each of the patent offices of the PRC, South Korea, Germany, Russian Federation, France, and Ukraine, all of the top five applicant institutions were from the home country. In contrast, all the top five applicant institutions in Australia's patent office came from the USA. Four out of the top five application institutions in both Canada's and Mexico's patent offices were from the USA. In addition, none of the top five applicant institutions in New Zealand's patent office was from its home country. Some internationally active applicant institutions that ranked among the top five in different countries'/regions' patent offices included IBM (from the US), the University of California (from the USA), Samsung Electronics Co. Ltd. (South Korea), Hon Hai Prec Ind Co. Ltd. (Taiwan), Industrial Technology Research Institute (Ind Tech Res Inst; Taiwan), Hyperion Catalysis International Inc. (USA), and General Electric (USA).

In the USA's patent office, IBM ranked first, followed by the University of California and Samsung Electronic Co. In Japan's patent office, the National Institute for Materials Science (Nat Inst for Materials Science) ranked first followed by the National Institute of Advanced Industrial Science and Technology (Nat Inst of Adv Ind \& Technol) and Matsushita 
Table 4 Top five applicant institutions in the 15 patent offices based on the number of nanotechnology patent applications from 1991 to 2008

\begin{tabular}{|c|c|c|c|c|c|c|c|}
\hline No. & $\begin{array}{l}\text { Patent office/ } \\
\text { repository }\end{array}$ & Rank & Applicant institution & $\begin{array}{l}\text { Country of } \\
\text { the institution }\end{array}$ & $\begin{array}{l}\text { Number of } \\
\text { nanotechnology } \\
\text { patent applications } \\
(1991-2008)\end{array}$ & 2000 & 2008 \\
\hline \multirow[t]{5}{*}{1} & \multirow[t]{5}{*}{ USA } & 1 & IBM & USA & 277 & 11 & 54 \\
\hline & & 2 & Univ California & USA & 209 & 11 & 29 \\
\hline & & 3 & Samsung Electronics Co. Ltd. & South Korea & 172 & 0 & 69 \\
\hline & & 4 & Hon Hai Prec Ind Co. Ltd. & Taiwan & 157 & 0 & 54 \\
\hline & & 5 & Ind Tech Res Inst & Taiwan & 106 & 3 & 15 \\
\hline \multirow[t]{5}{*}{2} & \multirow[t]{5}{*}{ PRC } & 1 & Chinese Academy of Science ${ }^{a}$ & PRC & 1,155 & 14 & 312 \\
\hline & & 2 & Univ Zhejiang & $\mathrm{PRC}$ & 464 & 3 & 129 \\
\hline & & 3 & Univ Tsinghua & PRC & 461 & 2 & 91 \\
\hline & & 4 & Univ Shanghai Jiaotong & PRC & 409 & 3 & 75 \\
\hline & & 5 & Univ Fudan & $\mathrm{PRC}$ & 317 & 3 & 81 \\
\hline \multirow[t]{5}{*}{3} & \multirow[t]{5}{*}{ Japan } & 1 & Nat Inst for Materials Science & Japan & 334 & 0 & 60 \\
\hline & & 2 & Nat Inst of Adv Ind \& Technol & Japan & 322 & 0 & 69 \\
\hline & & 3 & Matsushita Electric Ind Co. Ltd. & Japan & 263 & 6 & 37 \\
\hline & & 4 & Fujitsu Ltd. & Japan & 247 & 13 & 48 \\
\hline & & 5 & Canon Kk. & Japan & 222 & 11 & 26 \\
\hline \multirow[t]{5}{*}{4} & \multirow[t]{5}{*}{ South Korea } & 1 & Samsung Electronics Co. Ltd. & South Korea & 327 & 1 & 82 \\
\hline & & 2 & Korea Inst Science Technology & South Korea & 253 & 3 & 57 \\
\hline & & 3 & LG Electronics Inc. & South Korea & 153 & 2 & 26 \\
\hline & & 4 & Samsung Sdi Co. Ltd. & South Korea & 144 & 1 & 12 \\
\hline & & 5 & Seoul National University & South Korea & 120 & 0 & 46 \\
\hline \multirow[t]{5}{*}{5} & \multirow[t]{5}{*}{ Canada } & 1 & Xerox Co. & US & 27 & 0 & 18 \\
\hline & & 2 & Nantero Inc. & US & 25 & 0 & 0 \\
\hline & & 3 & Nat Res Council & Canada & 23 & 1 & 1 \\
\hline & & 4 & Hyperion Catalysis International Inc. & USA & 21 & 0 & 3 \\
\hline & & 5 & Nanosys Inc. & USA & 18 & 0 & 0 \\
\hline \multirow[t]{5}{*}{6} & \multirow[t]{5}{*}{ Taiwan $^{\mathrm{b}}$} & 1 & Ind Tech Res Inst & Taiwan & 201 & 0 & 23 \\
\hline & & 2 & Hon Hai Prec Ind Co. Ltd. & Taiwan & 78 & 0 & 51 \\
\hline & & 3 & Univ Nat Cheng Kung & Taiwan & 32 & 0 & 2 \\
\hline & & 4 & IBM & USA & 25 & 3 & 28 \\
\hline & & 5 & Univ Nat Chiao Tung & Taiwan & 17 & 0 & 3 \\
\hline \multirow[t]{5}{*}{7} & \multirow[t]{5}{*}{ Germany } & 1 & Infineon Technologies AG & Germany & 55 & 0 & 0 \\
\hline & & 2 & Fraunhofer Ges Forschung & Germany & 44 & 4 & 4 \\
\hline & & 3 & Siemens AG & Germany & 36 & 2 & 10 \\
\hline & & 4 & Henkel Kgaa & Germany & 31 & 5 & 0 \\
\hline & & 5 & Hahn Meitner Inst Berlin Gmbh & Germany & 20 & 0 & 0 \\
\hline \multirow[t]{5}{*}{8} & \multirow[t]{5}{*}{ Australia } & 1 & Univ California & US & 37 & 3 & 5 \\
\hline & & 2 & Univ Northwestern & US & 18 & 1 & 0 \\
\hline & & 3 & Hyperion Catalysis International Inc. & US & 16 & 3 & 4 \\
\hline & & 4 & Nanosphere Inc. & US & 15 & 0 & 1 \\
\hline & & 5 & Harvard College & US & 14 & 0 & 1 \\
\hline \multirow[t]{5}{*}{9} & \multirow[t]{5}{*}{ Russian Federation } & 1 & G Obrazovatel Noe Uchrezhdenie & Russian Fed. & 45 & 0 & 22 \\
\hline & & 2 & Zao NT MDT & Russian Fed. & 11 & 4 & 1 \\
\hline & & 3 & Boreskova Inst Kataliza Sibir & Russian Fed. & 10 & 1 & 3 \\
\hline & & 3 & Inst Fiz Tverdogo Tela Ran & Russian Fed. & 10 & 0 & 6 \\
\hline & & 3 & Inst Ehlektrofiziki Ural Skogo & Russian Fed. & 10 & 6 & 0 \\
\hline
\end{tabular}


Table 4 continued

\begin{tabular}{|c|c|c|c|c|c|c|c|}
\hline No. & $\begin{array}{l}\text { Patent office/ } \\
\text { repository }\end{array}$ & Rank & Applicant institution & $\begin{array}{l}\text { Country of } \\
\text { the institution }\end{array}$ & $\begin{array}{l}\text { Number of } \\
\text { nanotechnology } \\
\text { patent applications } \\
(1991-2008)\end{array}$ & 2000 & 2008 \\
\hline \multirow[t]{5}{*}{10} & \multirow[t]{5}{*}{ Mexico } & 1 & Procter \& Gamble & USA & 23 & 0 & 2 \\
\hline & & 2 & Elan Pharma International Ltd. & Ireland & 9 & 0 & 9 \\
\hline & & 2 & Hyperion Catalysis International Inc. & USA & 9 & 0 & 0 \\
\hline & & 4 & Kimberly Clark Co. & USA & 8 & 0 & 1 \\
\hline & & 4 & Rohm \& Haas & USA & 8 & 0 & 0 \\
\hline \multirow[t]{5}{*}{11} & \multirow[t]{5}{*}{ UK } & 1 & Toshiba Res Europ Ltd. & UK & 13 & 1 & 4 \\
\hline & & 2 & Hitachi Europ Ltd. & UK & 9 & 0 & 0 \\
\hline & & 3 & Gen Electric & USA & 8 & 1 & 0 \\
\hline & & 4 & Intel Co. & USA & 7 & 0 & 3 \\
\hline & & 5 & Waters Investments Ltd. & USA & 6 & 0 & 0 \\
\hline \multirow[t]{5}{*}{12} & \multirow[t]{5}{*}{ France } & 1 & Centre Nat Rech Scient & France & 58 & 0 & 5 \\
\hline & & 2 & Commissariat Energie Atomique & France & 41 & 1 & 3 \\
\hline & & 3 & O'real & France & 27 & 0 & 0 \\
\hline & & 4 & Rhone Poulenc Chimie & France & 10 & 0 & 0 \\
\hline & & 5 & Arkema Sa & France & 8 & 0 & 0 \\
\hline \multirow[t]{5}{*}{13} & \multirow[t]{5}{*}{ Brazil } & 1 & Unicamp & Brazil & 13 & 0 & 1 \\
\hline & & 2 & Comissao Nac de En Nuclear & Brazil & 8 & 0 & 1 \\
\hline & & 3 & Gen Electric & US & 6 & 0 & 2 \\
\hline & & 4 & Du Pont & US & 5 & 0 & 5 \\
\hline & & 5 & Gomes Uilame Umbelino & Brazil & 5 & 0 & 1 \\
\hline \multirow[t]{5}{*}{14} & \multirow[t]{5}{*}{ Ukraine } & 1 & Kaplunenko Volodymyr Heorhiiov & Ukraine & 99 & 0 & 53 \\
\hline & & 1 & Kosinov Mykola Vasyliovych & Ukraine & 99 & 0 & 53 \\
\hline & & 3 & Shulzhenko Oleksandr Oleksandr & Ukraine & 6 & 0 & 0 \\
\hline & & 4 & $\begin{array}{l}\text { Lytvynenko Yurii } \\
\text { Mykhailovych }\end{array}$ & Ukraine & 5 & 0 & 0 \\
\hline & & 5 & $\begin{array}{l}\text { Lviv Polytekhnika } \\
\text { Nat Universi }\end{array}$ & Ukraine & 4 & 0 & 1 \\
\hline \multirow[t]{5}{*}{15} & \multirow[t]{5}{*}{ New Zealand } & 1 & Eastman Kodak Co. & US & 4 & 0 & 0 \\
\hline & & 2 & Snow Brand Milk Prod Co. Ltd. & Japan & 3 & 1 & 0 \\
\hline & & 3 & Smithkline Beecham Co. & USA & 2 & 0 & 0 \\
\hline & & 3 & Technologies Avancees \& Membra & France & 2 & 0 & 0 \\
\hline & & 3 & Univ Johns Hopkins & USA & 2 & 0 & 0 \\
\hline
\end{tabular}

${ }^{a}$ In our data collection, Chinese Academy of Sciences had variations of its name in English and it also has several affiliated organizations. We manually checked and came up with 27 different institution names which are all essentially Chinese Academy of Sciences. The number reported in the table is the sum of all the nanotechnology patent applications published by these 27 institutions

b As noted previously, data from 2007 was used for Taiwan's patent office, rather than 2008

Electric Ind Co. Ltd. In PRC's patent offices, all the leading applicants are academic or academy research institutions.

Compared with 2000, there is a general increase in the number of nanotechnology patent applications published by the top institutions in 2008. Among the top five institutions, in each of the patent offices of the USA, PRC, and Australia, the institution with the largest numbers of nanotechnology patent applications from 1991 to 2008 also ranked first in 2000.

\section{Top technology fields}

We used the International Patent Classification (IPC) class instead of the European Patent Classification (EPC) class to indicate technology fields in Table 5 
Table 5 Top five technology fields in the 15 patent offices based on the number of nanotechnology patent applications from 1991 to 2008

\begin{tabular}{|c|c|c|c|c|c|c|c|}
\hline No & $\begin{array}{l}\text { Patent office/ } \\
\text { repository }\end{array}$ & Rank & IPC class & Class name & $\begin{array}{l}\text { Number of } \\
\text { nanotechnology patent } \\
\text { applications } \\
(1991-2008)\end{array}$ & 2000 & 2008 \\
\hline \multirow[t]{5}{*}{1} & \multirow[t]{5}{*}{ USA } & 1 & H01L & $\begin{array}{l}\text { Semiconductor devices; electric solid state devices } \\
\text { not otherwise provided for }\end{array}$ & 4,203 & 76 & 743 \\
\hline & & 2 & $\mathrm{~A} 61 \mathrm{~K}$ & Preparations for medical, dental, or toilet purposes & 1,974 & 51 & 367 \\
\hline & & 3 & G01N & $\begin{array}{l}\text { Investigating or analyzing materials by determining } \\
\text { their chemical or physical properties }\end{array}$ & 1,754 & 36 & 230 \\
\hline & & 4 & $\mathrm{C} 01 \mathrm{~B}$ & Non-metallic elements; compounds thereof & 1,453 & 23 & 187 \\
\hline & & 5 & B32B & $\begin{array}{l}\text { Layered products, i.e., products built-up of strata of } \\
\text { flat or non-flat, e.g., cellular or honeycomb }\end{array}$ & 1,400 & 15 & 444 \\
\hline \multirow[t]{5}{*}{2} & \multirow[t]{5}{*}{ PRC } & 1 & A61K & Preparations for medical, dental, or toilet purposes & 1,549 & 9 & 370 \\
\hline & & 2 & $\mathrm{C} 01 \mathrm{~B}$ & Non-metallic elements; compounds thereof & 1,501 & 14 & 392 \\
\hline & & 3 & B01J & $\begin{array}{l}\text { Chemical or physical processes, e.g., catalysis, } \\
\text { colloid chemistry; their relevant apparatus }\end{array}$ & 1,311 & 11 & 388 \\
\hline & & 4 & $\mathrm{C} 08 \mathrm{~L}$ & Compositions of macromolecular compounds & 1,247 & 7 & 349 \\
\hline & & 5 & H01L & $\begin{array}{l}\text { Semiconductor devices; electric solid state devices } \\
\text { not otherwise provided for }\end{array}$ & 1,095 & 4 & 350 \\
\hline \multirow[t]{5}{*}{3} & \multirow[t]{5}{*}{ Japan } & 1 & H01L & $\begin{array}{l}\text { Semiconductor devices; electric solid state devices } \\
\text { not otherwise provided for }\end{array}$ & 2,324 & 81 & 367 \\
\hline & & 2 & $\mathrm{C} 01 \mathrm{~B}$ & Non-metallic elements; compounds thereof & 1,994 & 55 & 292 \\
\hline & & 3 & $\mathrm{~B} 82 \mathrm{~B}$ & Nano-structures manufacture or treatment thereof & 1,599 & 35 & 229 \\
\hline & & 4 & G01N & $\begin{array}{l}\text { Investigating or analyzing materials by determining } \\
\text { their chemical or physical properties }\end{array}$ & 1,123 & 47 & 89 \\
\hline & & 5 & H01J & Electric discharge tubes or discharge lamps & 1,031 & 58 & 82 \\
\hline \multirow[t]{5}{*}{4} & \multirow[t]{5}{*}{ South Korea } & 1 & $\mathrm{~B} 82 \mathrm{~B}$ & Nano-structures manufacture or treatment thereof & 1,280 & 5 & 417 \\
\hline & & 2 & H01L & $\begin{array}{l}\text { Semiconductor devices; electric solid state devices } \\
\text { not otherwise provided for }\end{array}$ & 1,094 & 29 & 238 \\
\hline & & 3 & $\mathrm{C} 01 \mathrm{~B}$ & Non-metallic elements; compounds thereof & 409 & 5 & 103 \\
\hline & & 4 & $\mathrm{C} 08 \mathrm{~K}$ & $\begin{array}{l}\text { Use of inorganic or non-macromolecular organic } \\
\text { substances as compounding ingredients }\end{array}$ & 374 & 0 & 88 \\
\hline & & 5 & H01J & Electric discharge tubes or discharge lamps & 361 & 7 & 30 \\
\hline \multirow[t]{5}{*}{5} & \multirow[t]{5}{*}{ Canada } & 1 & A61K & Preparations for medical, dental, or toilet purposes & 328 & 8 & 47 \\
\hline & & 2 & G01N & $\begin{array}{l}\text { Investigating or analyzing materials by determining } \\
\text { their chemical or physical properties }\end{array}$ & 169 & 7 & 9 \\
\hline & & 3 & B01J & $\begin{array}{l}\text { Chemical or physical processes, e.g., catalysis, } \\
\text { colloid chemistry; their relevant apparatus }\end{array}$ & 148 & 10 & 8 \\
\hline & & 4 & $\mathrm{C} 01 \mathrm{~B}$ & Non-metallic elements; compounds thereof & 144 & 2 & 23 \\
\hline & & 5 & H01L & $\begin{array}{l}\text { Semiconductor devices; electric solid state devices } \\
\text { not otherwise provided for }\end{array}$ & 123 & 4 & 22 \\
\hline \multirow[t]{5}{*}{6} & \multirow[t]{5}{*}{ Taiwan $^{\mathrm{a}}$} & 1 & H01L & $\begin{array}{l}\text { Semiconductor devices; electric solid state devices } \\
\text { not otherwise provided for }\end{array}$ & 367 & 11 & 47 \\
\hline & & 2 & $\mathrm{C} 01 \mathrm{~B}$ & Non-metallic elements; compounds thereof & 114 & 1 & 14 \\
\hline & & 3 & H01J & Electric discharge tubes or discharge lamps & 112 & 1 & 9 \\
\hline & & 4 & $\mathrm{C} 23 \mathrm{C}$ & $\begin{array}{l}\text { Coating metallic material coating material with } \\
\text { metallic material surface treatment of metallic } \\
\text { material by diffusion into the surface, by } \\
\text { chemical conversion or substitution coating by } \\
\text { vacuum evaporation, by sputtering, by ion } \\
\text { implantation or by chemical vapor deposition, in } \\
\text { general }\end{array}$ & 78 & 2 & 13 \\
\hline & & 5 & G01N & $\begin{array}{l}\text { Investigating or analyzing materials by determining } \\
\text { their chemical or physical properties }\end{array}$ & 71 & 0 & 15 \\
\hline
\end{tabular}


Table 5 continued

\begin{tabular}{|c|c|c|c|c|c|c|c|}
\hline No & $\begin{array}{l}\text { Patent office/ } \\
\text { repository }\end{array}$ & Rank & IPC class & Class name & $\begin{array}{l}\text { Number of } \\
\text { nanotechnology patent } \\
\text { applications } \\
(1991-2008)\end{array}$ & 2000 & 2008 \\
\hline \multirow[t]{5}{*}{7} & \multirow[t]{5}{*}{ Australia } & 1 & A61K & Preparations for medical, dental, or toilet purposes & 295 & 22 & 19 \\
\hline & & 2 & $\mathrm{C} 01 \mathrm{~B}$ & Non-metallic elements; compounds thereof & 212 & 12 & 7 \\
\hline & & 3 & H01L & $\begin{array}{l}\text { Semiconductor devices; electric solid state devices } \\
\text { not otherwise provided for }\end{array}$ & 209 & 9 & 11 \\
\hline & & 4 & B01J & $\begin{array}{l}\text { Chemical or physical processes, e.g., catalysis, } \\
\text { colloid chemistry; their relevant apparatus }\end{array}$ & 177 & 10 & 12 \\
\hline & & 5 & G01N & $\begin{array}{l}\text { Investigating or analyzing materials by determining } \\
\text { their chemical or physical properties }\end{array}$ & 163 & 15 & 4 \\
\hline \multirow[t]{5}{*}{8} & \multirow[t]{5}{*}{ Germany } & 1 & H01L & $\begin{array}{l}\text { Semiconductor devices; electric solid state devices } \\
\text { not otherwise provided for }\end{array}$ & 165 & 7 & 17 \\
\hline & & 2 & B01J & $\begin{array}{l}\text { Chemical or physical processes, e.g., catalysis, } \\
\text { colloid chemistry; their relevant apparatus }\end{array}$ & 135 & 13 & 10 \\
\hline & & 3 & B82B & Nano-structures manufacture or treatment thereof & 121 & 3 & 16 \\
\hline & & 4 & G01N & $\begin{array}{l}\text { Investigating or analyzing materials by determining } \\
\text { their chemical or physical properties }\end{array}$ & 111 & 3 & 13 \\
\hline & & 5 & A61K & Preparations for medical, dental, or toilet purposes & 103 & 6 & 8 \\
\hline \multirow[t]{5}{*}{9} & \multirow{5}{*}{$\begin{array}{l}\text { Russian } \\
\text { Federation }\end{array}$} & 1 & $\mathrm{~B} 82 \mathrm{~B}$ & Nano-structures manufacture or treatment thereof & 118 & 2 & 55 \\
\hline & & 2 & $\mathrm{H} 01 \mathrm{~L}$ & $\begin{array}{l}\text { Semiconductor devices; electric solid state devices } \\
\text { not otherwise provided for }\end{array}$ & 88 & 4 & 13 \\
\hline & & 3 & $\mathrm{C} 01 \mathrm{~B}$ & Non-metallic elements; compounds thereof & 75 & 2 & 16 \\
\hline & & 4 & B01J & $\begin{array}{l}\text { Chemical or physical processes, e.g., catalysis, } \\
\text { colloid chemistry; their relevant apparatus }\end{array}$ & 69 & 6 & 15 \\
\hline & & 5 & A61K & Preparations for medical, dental, or toilet purposes & 58 & 1 & 12 \\
\hline \multirow[t]{5}{*}{10} & \multirow[t]{5}{*}{ UK } & 1 & H01L & $\begin{array}{l}\text { Semiconductor devices; electric solid state devices } \\
\text { not otherwise provided for }\end{array}$ & 83 & 2 & 16 \\
\hline & & 2 & G01N & $\begin{array}{l}\text { Investigating or analyzing materials by determining } \\
\text { their chemical or physical properties }\end{array}$ & 58 & 2 & 15 \\
\hline & & 3 & B01D & Separation & 30 & 2 & 2 \\
\hline & & 4 & A61K & Preparations for medical, dental, or toilet purposes & 29 & 0 & 7 \\
\hline & & 5 & $\mathrm{C} 01 \mathrm{~B}$ & Non-metallic elements; compounds thereof & 28 & 0 & 2 \\
\hline \multirow[t]{5}{*}{11} & \multirow[t]{5}{*}{ Mexico } & 1 & A61K & Preparations for medical, dental, or toilet purposes & 109 & 0 & 26 \\
\hline & & 2 & B01J & $\begin{array}{l}\text { Chemical or physical processes, e.g., catalysis, } \\
\text { colloid chemistry; their relevant apparatus }\end{array}$ & 60 & 0 & 0 \\
\hline & & 3 & $\mathrm{C} 08 \mathrm{~K}$ & $\begin{array}{l}\text { Use of inorganic or non-macromolecular organic } \\
\text { substances as compounding ingredients }\end{array}$ & 58 & 0 & 8 \\
\hline & & 4 & C08L & Compositions of macromolecular compounds & 52 & 0 & 3 \\
\hline & & 5 & C09D & $\begin{array}{l}\text { Coating compositions, e.g. paints, varnishes, } \\
\text { lacquers; filling-pastes; chemical paint or ink } \\
\text { removers; inks; correcting fluids; wood stains; } \\
\text { pastes or solids for coloring or printing; use of } \\
\text { materials therefore }\end{array}$ & 45 & 0 & 8 \\
\hline \multirow[t]{5}{*}{12} & \multirow[t]{5}{*}{ France } & 1 & A61K & Preparations for medical, dental, or toilet purposes & 69 & 0 & 2 \\
\hline & & 2 & H01L & $\begin{array}{l}\text { Semiconductor devices; electric solid state devices } \\
\text { not otherwise provided for }\end{array}$ & 61 & 0 & 6 \\
\hline & & 3 & B82B & Nano-structures manufacture or treatment thereof & 55 & 0 & 7 \\
\hline & & 4 & $\mathrm{C} 01 \mathrm{~B}$ & Non-metallic elements; compounds thereof & 47 & 0 & 7 \\
\hline & & 5 & A61Q & Use of cosmetics or similar toilet preparations & 45 & 0 & 2 \\
\hline
\end{tabular}


Table 5 continued

\begin{tabular}{|c|c|c|c|c|c|c|c|}
\hline No & $\begin{array}{l}\text { Patent office/ } \\
\text { repository }\end{array}$ & Rank & IPC class & Class name & $\begin{array}{l}\text { Number of } \\
\text { nanotechnology patent } \\
\text { applications } \\
\text { (1991-2008) }\end{array}$ & 2000 & 2008 \\
\hline \multirow[t]{5}{*}{13} & \multirow[t]{5}{*}{ Brazil } & 1 & A61K & Preparations for medical, dental, or toilet purposes & 65 & 0 & 21 \\
\hline & & 2 & $\mathrm{C} 08 \mathrm{~K}$ & $\begin{array}{l}\text { Use of inorganic or non-macromolecular organic } \\
\text { substances as compounding ingredients }\end{array}$ & 30 & 0 & 7 \\
\hline & & 3 & C08L & Compositions of macromolecular compounds & 28 & 0 & 6 \\
\hline & & 4 & B01J & $\begin{array}{l}\text { Chemical or physical processes, e.g., catalysis, } \\
\text { colloid chemistry; their relevant apparatus }\end{array}$ & 28 & 0 & 8 \\
\hline & & 5 & $\mathrm{~B} 82 \mathrm{~B}$ & Nano-structures manufacture or treatment thereof & 24 & 0 & 12 \\
\hline \multirow[t]{5}{*}{14} & \multirow[t]{5}{*}{ Ukraine } & 1 & B01J & $\begin{array}{l}\text { Chemical or physical processes, e.g., catalysis, } \\
\text { colloid chemistry; their relevant apparatus }\end{array}$ & 52 & 0 & 25 \\
\hline & & 2 & $\mathrm{C} 01 \mathrm{~B}$ & Non-metallic elements; compounds thereof & 24 & 0 & 3 \\
\hline & & 3 & $\mathrm{~B} 22 \mathrm{~F}$ & $\begin{array}{l}\text { Working metallic powder; manufacture of articles } \\
\text { from metallic powder; making metallic powder }\end{array}$ & 21 & 0 & 4 \\
\hline & & 4 & $\mathrm{C} 12 \mathrm{~N}$ & Micro-organisms or enzymes; compositions thereof & 19 & 0 & 16 \\
\hline & & 5 & $\mathrm{C} 02 \mathrm{~F}$ & Treatment of water, waste water, sewage, or sludge & 18 & 0 & 4 \\
\hline \multirow[t]{5}{*}{15} & \multirow[t]{5}{*}{ New Zealand } & 1 & A61K & Preparations for medical, dental, or toilet purposes & 62 & 4 & 8 \\
\hline & & 2 & A61P & $\begin{array}{l}\text { Therapeutic activity of chemical compounds or } \\
\text { medicinal preparations }\end{array}$ & 28 & 1 & 3 \\
\hline & & 3 & B01J & $\begin{array}{l}\text { Chemical or physical processes, e.g., catalysis, } \\
\text { colloid chemistry; their relevant apparatus }\end{array}$ & 20 & 2 & 4 \\
\hline & & 4 & B01D & Separation & 18 & 1 & 3 \\
\hline & & 5 & $\mathrm{C} 07 \mathrm{~K}$ & Peptides & 17 & 0 & 3 \\
\hline
\end{tabular}

a As noted previously, data from 2007 was used for Taiwan's patent office, rather than 2008

because the EPC class information is incomplete in some patent offices (repositories). Among the top five technology fields in the 15 patent offices, there were 19 unique IPC classes, 10 of which ranked among the top five in more than one patent office:

- "Semiconductor devices; electric solid state devices not otherwise provided for" (H01L) ranked among the top five in 11 patent offices (except in those of Mexico, Brazil, the Ukraine, and New Zealand)

- "Preparations for medical, dental, or toilet purposes" (A61K) ranked among the top five in 11 patent offices (except in those of Japan, South Korea, Taiwan, and the Ukraine)

- "Non-metallic elements; compounds thereof" (C01B) ranked among the top five in 11 patent offices (except in those of Germany, Mexico, Brazil, and New Zealand)

- "Chemical or physical processes, e.g., catalysis, colloid chemistry; their relevant apparatus" (B01J) also ranked among the top five in nine patent offices
- "Investigating or analyzing materials by determining their chemical or physical properties" (G01N) ranked among the top five in seven patent offices

- "Nano-structures manufacture or treatment thereof" (B82B) ranked among the top five in six patent offices.

In the USPTO, "Semiconductor devices; electric solid state devices not otherwise provided for" (H01L) ranked first, followed by "Preparations for medical, dental, or toilet purposes" (A61K). Such rankings are consistent with the results reported in our previous study for granted patents. In addition, "Investigating or analyzing materials by determining their chemical or physical properties" (G01N) and "Layered products, i.e., products built-up of strata of flat or non-flat, e.g., cellular or honeycomb" (B32B), which ranked third and fifth, respectively, in this study, ranked fifth and fourth, respectively, in the previous study ( $\mathrm{Li}$ et al. 2007). However, "Non-metallic elements; compounds thereof" (C01B), which was also among the top five, did not appear among the top 10 technology fields as reported by Li et al. (2007). 
In Japan's patent office, "Semiconductor devices; electric solid state devices not otherwise provided for" (H01L) ranked first, followed by "Non-metallic elements; compounds thereof" (C01B), "Nano-structures manufacture or treatment thereof" (B82B), "Investigating or analyzing materials by determining their chemical or physical properties" (G01N), and "Electric discharge tubes or discharge lamps" (H01J). All these technology fields ranked among the top 10 in the previous study ( $\mathrm{Li}$ et al. 2007). Except "Nano-structures manufacture or treatment thereof" (B82B), which ranked eighth in Li et al. (2007), they all ranked among the top five as well.

Compared to 2000 , there were many more nanotechnology patent applications in the top five technology fields in 2008 for different patent offices, including the patent offices of the USA, PRC, Japan, South Korea, Canada, Germany, Russian Federation, the UK, Mexico, France, Brazil, the Ukraine, and New Zealand. Since the patent offices of Mexico, Brazil, and Ukraine did not have nanotechnology patent applications in 2000, there were no applications from their top five technology fields in 2000. In addition, none of the eight applications in France's patent office in 2000 belonged to its top five technology fields. In 2008, almost all the top five technology fields in each of the 15 patent offices had nanotechnology patent applications.

For the patent offices of the USA, Japan, Taiwan, Australia, and New Zealand, the technology field that ranked the first in each of them based on data from 1991 to 2008 also had the largest number of nanotechnology patent applications in 2000. In 2008, there were 13 patent offices (excepting the patent offices of PRC and France) for which the technology field which ranked first based on data from 1991 to 2008, also had the largest number of nanotechnology patent applications in 2008 (Taiwan in 2007).

\section{Topic analysis}

Content maps were used to visualize the major technology topics in different patent offices (repositories). Since the patent offices of the USA and PRC had many more nanotechnology patent applications than other countries, we created content maps for both of them. In order to get a better understanding of the topic evolution for all the 15 patent offices, we also created content maps using the data from all the 15 patent offices for years 2000 and 2008.

We used the multi-level self-organization map algorithm (Chen et al. 1996; Ong et al. 2005) developed by the Artificial Intelligence Lab at the University of Arizona. A content map has two components: a folder tree, and a hierarchical map. Each node in the folder tree, corresponding to a region in the hierarchical map, is a topic (keyword) identified from the document. Conceptually closer technology topics are positioned closer geographically. The numbers of documents assigned to the different levels of topics are presented after the topic labels. The size of each topic region also generally corresponds to the number of documents assigned to the topic. For each topic region, a growth rate is computed as the ratio between the number of documents in the current time period and that of the previous time period. A baseline growth rate is computed as the ratio between the total number of documents in the current time period and that of the previous time period. A topic region with a growth rate similar to the base growth rate is assigned a green color. A topic region with a higher or lower growth rate is assigned a warmer or colder color, respectively. If the topic is brand new, a red color is assigned to the region.

Figures 5, 6, and 7 show the content maps for the patent office of the USA in 2008 for USPTO, PRC, and for all the 15 patent offices, respectively. The data in 2008 for USPTO and all the 15 countries are compared to data in 2000 . For the patent office of the PRC, the 2008 data cannot be statistically well compared to data in 2000 , because the number of nanotechnology patent applications in 2000 is too limited to generate a content map at that date.

Compared with 2000, the nanotechnology patent applications published in the USPTO in 2008 (Fig. 5) have a baseline growth rate of 16.14 times, indicating a significant increase in nanotechnology research. Topics that appear in both years are: "Aqueous solutions," "Dielectric layers," and "Metal oxides," each of which had many more applications in 2008 than in 2000.

Topics which appeared in 2000 only included:

- Nanomaterial-related topics, such as "Carbon atoms," "Carbon nanotubes," and "Memory cells"

- Properties of nanomaterials, such as "Average molecular weight," "Low dielectric," "Molecular weights," and "Surface roughness" 


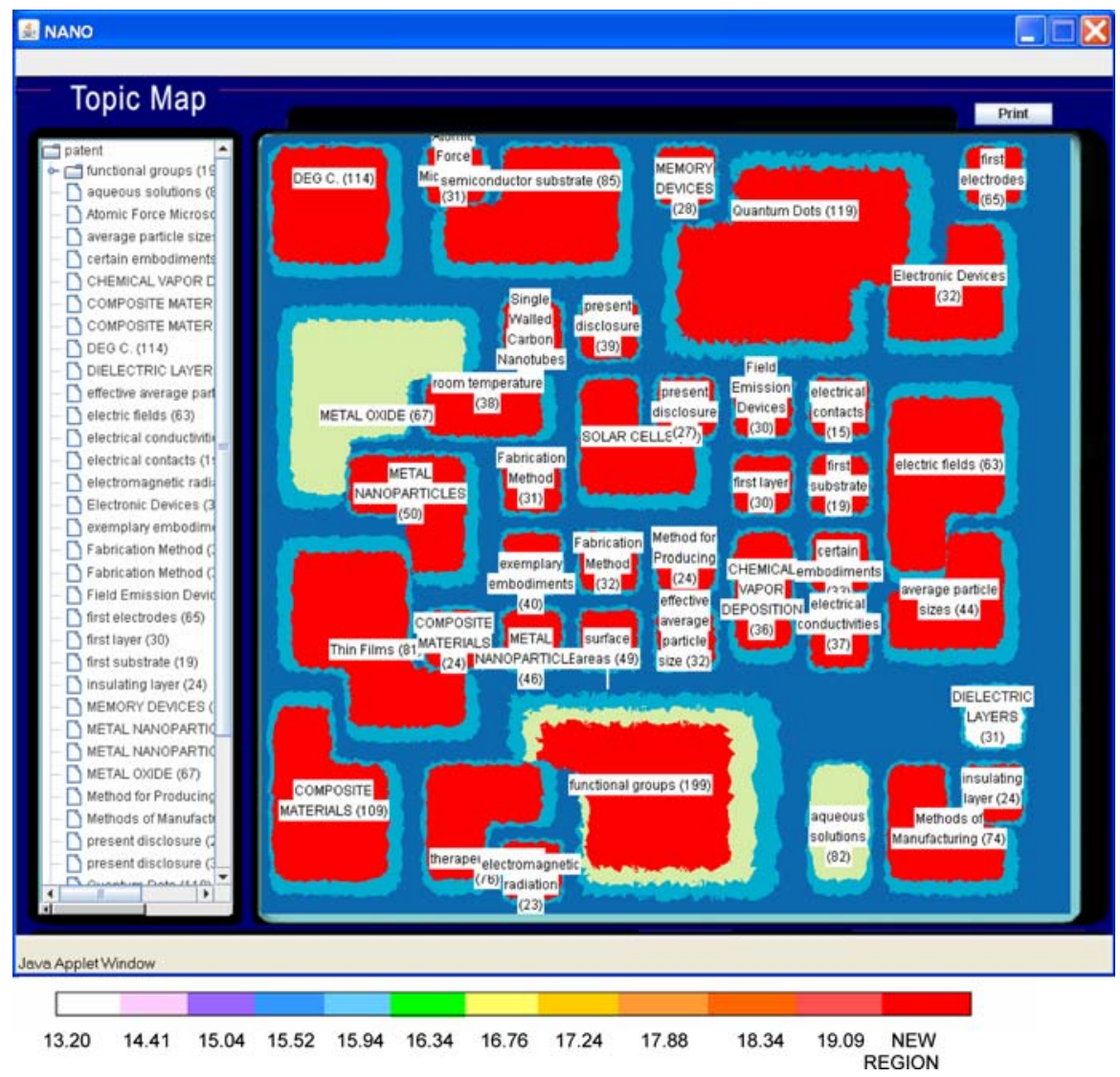

Fig. 5 The content map for patent applications at the USPTO in 2008 (the color means the rate of increase as compared to 2000; the "new region" in red is $92 \%$ )

- Nano-device related topics, such as "Alkali metals," "Laser beams," "Light source," "Magnetic recording medium," "Silicon substrates," "Substrate surfaces"

- Measurement- and method-related topics, such as "Nanometers at reaction conditions," "Surface roughness," and "Ultraviolet radiation."

The newly emerging topics of 2008 included:

- Nanomaterial-related topics, such as "Composite materials," "Metal nanoparticles," "Quantum dots," "Single walled carbon nanotubes," "Solar cells," and "Thin films"

- Properties of nanomaterials, such as "Average particle sizes," "Effective average particle size," and "Electrical conductivities"

- Nano-device related topics, such as "Electric fields," "Electronic devices," "Field emission devices," "Insulating layer," "Memory devices," "Semiconductor substrate," "Therapeutic agents"
- Measurement- and method-related topics, such as "Atomic force microscope," "Chemical vapor deposition," "Electromagnetic radiation," and "Fabrication method."

As shown in Fig. 6, the largest topic in the patent office of the PRC in 2008 was "human bodies" with 295 nanotechnology patent applications. Topics related to nanomaterials included "Carbon nanotube," "Composite materials," "Nanometer materials," "Quantum dots," and "Thin films." Topics related to properties of nanomaterials were "Good stability," "Grain diameter," "High activity," "High sensitivity," "Molar ratio," "pH values," "Service life," and "Weight percentages." Topics related to nano-device included "Deionized water," "Organic solvents," and "Stainless steels." Topics related to measurements and methods included "Aqueous solutions," "Convenient operation," "Production method," and "Water solution." 
Fig. 6 The content map for the patent office of the PRC in 2008

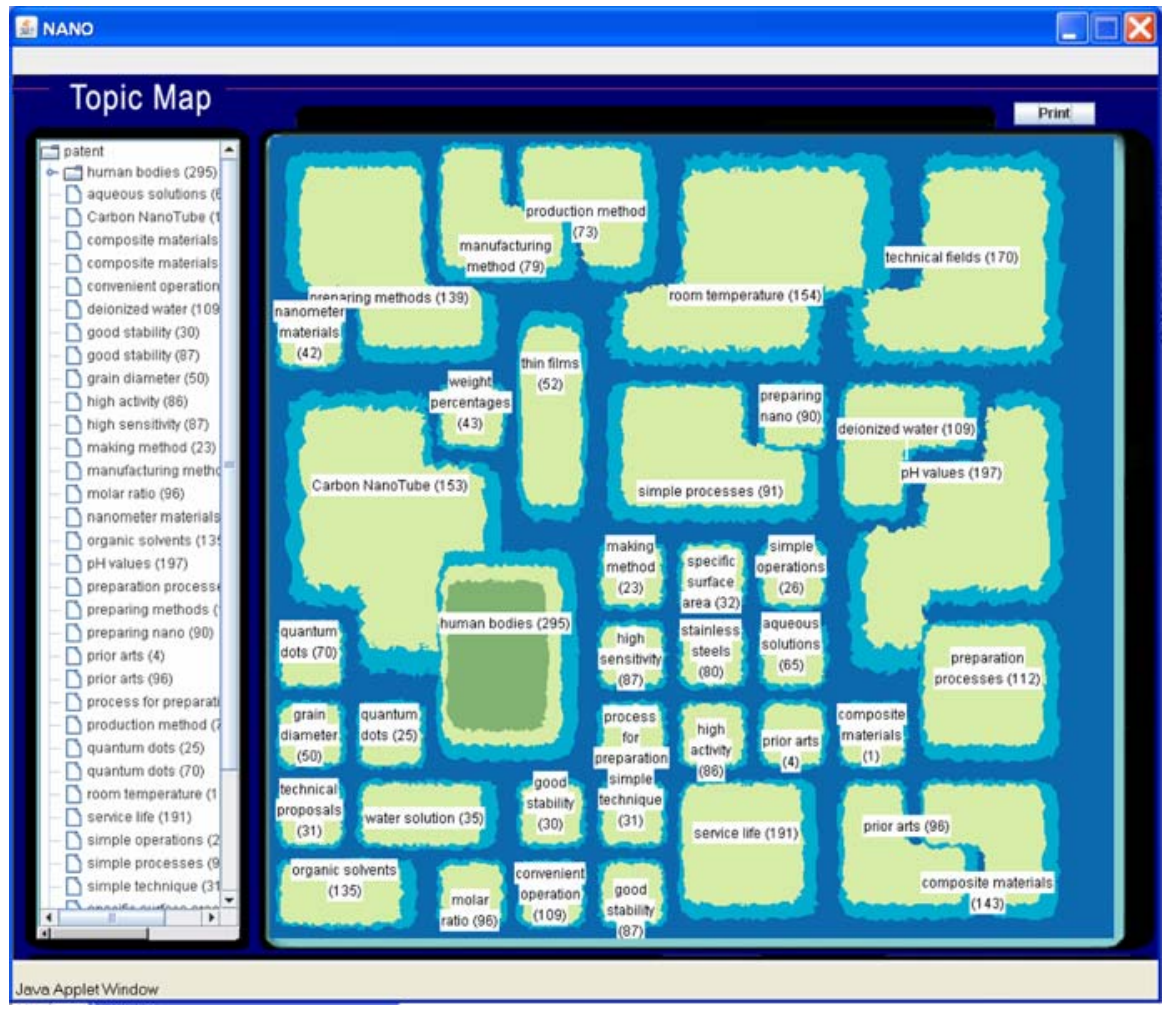

Compared with 2000 the nanotechnology patent applications published in all, the 15 patent offices in 2008 (Fig. 7) had a baseline growth rate of 12.57 , indicating a significant increase in nanotechnology research. Six main topics that appeared in both years included:

- "Average particle size"

- "Aqueous solutions"

- "Metal oxides"

- "Manufacturing method"

- "Thin films"

- "Functional groups"

Each of these topics had significantly more applications in 2008 than in 2000.

Older main topics that appeared in 2000 only included:

- Nanomaterial-related topics, such as "Carbon nanotube," "Composite materials," "Organic polymer," "Quantum dot," and "Such films"

- Properties of nanomaterials, such as "Average pore diameter," “Average size," "High densities," "Molecular weights," "Surface roughness," "Weight ratio," etc.
- Nano-device related topics, such as "Electric fields," "Electron beams," "Laser beams," "Light source," "Magnetic cores," "Semiconductor substrates," and "Transition metals"

- Measurement- and method-related topics, such as "Atomic force microscope," "Electromagnetic radiation," and "Ultraviolet radiation"

The newly emerging topics for 2008 included:

- Nanomaterial-related topics, such as "Composite materials," "Metal nanoparticles," and "Quantum dots"

- Properties of nanomaterials, such as "pH values," "High purities," and "Particle diameters"

- Nano-device related topics, such as "Organic solvents," "Semiconductor Devices," "Deionized water," and "Gate electrodes"

There were no new topics related to certain measurements or methods.

Patent family analysis

The patent office of each country or region has the jurisdiction to grant a patent for its own geographic 
area only. In order to achieve broader coverage of the exclusive rights for an invention, some patent applications are filed in multiple countries'/regions' patent offices, and, thus, become a patent family. All the patent applications (or granted patents) in a given patent family are equivalents and considered to be one invention.

\section{Patent family analysis within each patent office}

Table 6 lists the numbers of nanotechnology patent applications published in single patent office, two or more patent offices, and three or more patent offices. For example, 2,939 patent applications that were published in the US patent office had been also published in at least one other patent office. Among those patent applications, 741 had been published in three or more countries'/regions' patent offices. The patent offices of Japan, the PRC, and South Korea also had relatively larger numbers of nanotechnology patent applications published in multiple patent offices.

For each patent office, we also identified other patent offices with which it shared the greatest numbers of nanotechnology patent applications for the interval between 1991 and 2008. For example,
- The top five patent offices sharing nanotechnology patent applications with the USPTO were Japan (1,258), PRC (725); South Korea (636), Taiwan (353), and Canada (350). Our analysis shows that all other patent offices (except for Brazil's patent offices) shared the largest numbers of nanotechnology patent applications with the USPTO.

- The top five patent offices sharing nanotechnology patent applications with the PRC repository were those of the USA (725), South Korea (624), Japan (416), Taiwan (68), and Canada (40).

- The top five patent offices sharing nanotechnology patent applications with the JPO were those of the USA (1258), South Korea (450), PRC (416), Taiwan (107), and Canada (106).

\section{Patent family analysis across patent offices}

The network in Fig. 8 shows patent families across patent offices. In the network, nodes represent different countries'/regions' patent offices. The bigger the node, the higher the number of nanotechnology patent applications it shares with other countries'/regions' patent offices. The number of nanotechnology patent applications each patent office shared with others is

Table 6 Numbers of nanotechnology patent applications published in single patent office, two or more patent offices, and three or more patent offices (1991-2008)

\begin{tabular}{llllll}
\hline No. & $\begin{array}{l}\text { Patent office } \\
\text { (repository) }\end{array}$ & $\begin{array}{l}\text { No. of patent } \\
\text { applications } \\
\text { published in total }\end{array}$ & $\begin{array}{l}\text { No. of patent } \\
\text { applications published } \\
\text { in a single patent office }\end{array}$ & $\begin{array}{l}\text { No. of patent applications } \\
\text { published in } \geq 2 \text { patent } \\
\text { offices }\end{array}$ & $\begin{array}{l}\text { No. of patent applications } \\
\text { published in } \geq 3 \text { patent } \\
\text { offices }\end{array}$ \\
\hline 1 & USA & 19,665 & 16,726 & 2,939 & 741 \\
2 & PRC & 18,438 & 17,079 & 1,359 & 490 \\
3 & Japan & 10,763 & 9,084 & 1,679 & 614 \\
4 & South Korea & 5,963 & 4,731 & 1,232 & 491 \\
5 & Canada & 1,539 & 988 & 551 & 160 \\
6 & Taiwan & 1,363 & 900 & 463 & 123 \\
7 & Australia & 1,312 & 926 & 386 & 91 \\
8 & Germany & 1,296 & 1,229 & 67 & 21 \\
9 & Russian Federation & 859 & 785 & 74 & 31 \\
10 & Mexico & 471 & 228 & 243 & 96 \\
11 & UK & 412 & 291 & 121 & 32 \\
12 & France & 390 & 339 & 51 & 70 \\
13 & Brazil & 315 & 167 & 148 & 6 \\
14 & Ukraine & 243 & 231 & 12 & 38 \\
15 & New Zealand & 140 & 68 & 72 & \\
\hline
\end{tabular}


Fig. 7 The content map for all 15 patent offices in 2008 (the color means the rate of increase as compared to 2000; the "new region" in red is $68 \%$ )

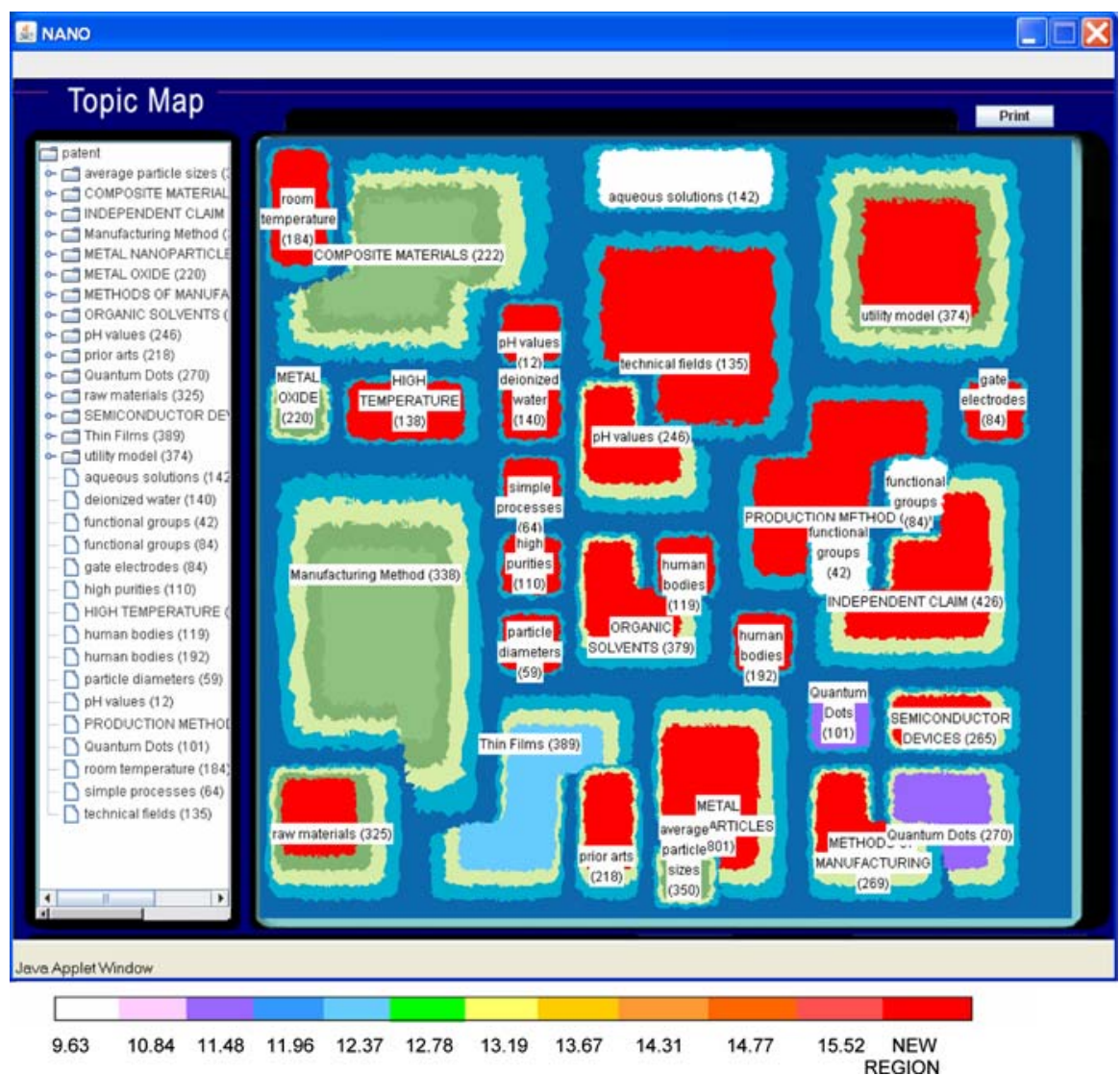

presented in parentheses after the node label. The link between two patent offices means that they have published nanotechnology patent applications in the same patent families. The thicker the link, the greater the number of nanotechnology patent applications the two patent offices shared. The number beside the link indicates how many nanotechnology patent applications the two patent offices shared. The network was drawn using open source software, NetDraw (available at: http://www.analytictech.com/Netdraw/netdraw.htm/).

Each of the top 15 patent offices shared nanotechnology patent applications with other patent offices. The USPTO shared the largest number of nanotechnology patent applications with others $(2,939)$, followed by the patent offices of Japan, PRC, and South Korea $(1,679 ; 1,359$; and 1,232 nanotechnology patent applications, respectively). The thickest link in the network shows that the patent offices of the US and Japan shared the largest number of nanotechnology patent applications (i.e., 1,258 applications).
We have identified 12 patent families that had equivalent nanotechnology patent applications published in at least five patent offices. The titles of the equivalent patents may or may not be the same.

\section{Conclusions}

The nanotechnology patent applications published in different countries'/regions' patent offices have been evaluated by using the esp@cenet "worldwide" database. Key findings from the longitudinal analysis of nanotechnology patent applications between 1991 and 2008 are:

- The worldwide growth rate of the number of nanotechnology patent applications between 2000 and 2008 is about $34.5 \%$ (Fig. 1). This rate is larger than the corresponding rate of increase for International Citation Index articles of about $25 \%$. The baseline growth rates of the number of patent applications for continuing 
Fig. 8 Patent families across leading 15 patent offices for nanotechnology applications from 1991 to 2008

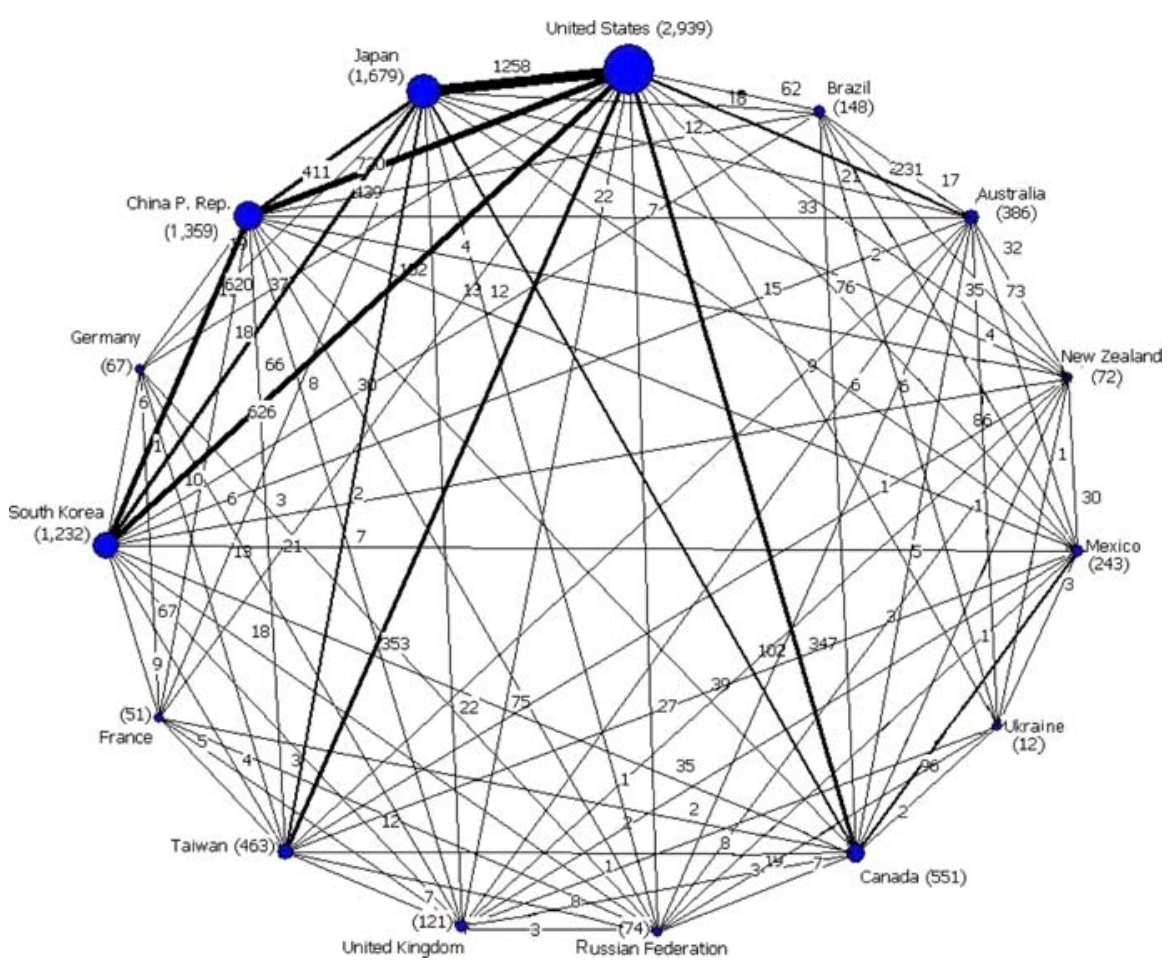

topics are 16.14 and 12.57 times in the interval from 1991 to 2008 for the USPTO and the top 15 nanotechnology patent repositories, respectively. The new nanotechnology topics in 2008 as compared with 2000 represent $92 \%$ in the USA (Fig. 5) and 68\% for top 15 repositories (Fig. 7). The baseline growth rate is significant in the PRC patent office, but the data available in 2000 are too limited to generate a content map in that year for comparison with 2008. The largest number of nanotechnology patent applications, as well as of the patent application families, are at the patent offices of the USA, PRC, Japan, and South Korea.

- A higher number of nanotechnology patent applications are published by applicants from their own countries/regions, indicating significant "home advantage" effects. The USA, Japan, Germany, South Korea, and France were the largest contributors in patent offices other than its repository. The top 15 patent offices except for Brazil's patent office shared the largest numbers of nanotechnology patent applications with the USPTO. Japan is the USPTO's largest partner cosharing 1,258 nanotechnology patent applications.
- Applicant institutions with large international activity are illustrated by IBM (from the USA), the University of California (from the USA), Samsung Electronics Co. Ltd. (from South Korea), Hon Hai Prec Ind Co. Ltd. (from Taiwan), and Industrial Technology Research Institute (Ind Tech Res Inst; from Taiwan), Hyperion Catalysis International Inc. (from the USA), and General Electric (Gen Electric, from the USA).

- The ranking of the most productive institutions and the categories of the lead technology fields in patent repositories have had relatively small changes over time, and few institutions or categories of technology fields were able to break into the top ranks. However, specific topics within various technology field categories changed rapidly after 2000. Topics that increased in 2008 in most of the 15 patent offices included: "Composite materials," "Deionized water," "Gate electrodes," "High purities," "Metal nanoparticles," "Organic solvents," "Particle diameters," "PH values," "Quantum dots," and "Semiconductor Devices."

- Several top technology fields (represented by IPC class) were shared by multiple repositories. 
"Semiconductor devices; electric solid state devices not otherwise provided for" (H01L) was among the top five technology fields in 11 out of the 15 patent offices. The following fields ranked among the top five in multiple repositories: "Preparations for medical, dental, or toilet purposes" (A61K), "Non-metallic elements; compounds thereof" (C01B), "Chemical or physical processes, e.g., catalysis, colloid chemistry; their relevant apparatus" (B01J), "Investigating or analyzing materials by determining their chemical or physical properties" (G01N), and "Nano-structures manufacture or treatment thereof" (B82B).

Acknowledgments This research was supported by the following awards: National Science Foundation: "Intelligent Patent Analysis for Nanoscale Science and Engineering," IIS0311652; "Mapping Nanotechnology Development," DMI0533749; and "Worldwide Nanotechnology Development: A Comparative Study of Global Patents" CMMI-0654232. The last co-author was supported by the Directorate for Engineering, NSF. We would like to thank esp@cenet for making the "worldwide" database available for research.

\section{References}

Albert R, Barabasi AL (2002) Statistical mechanics of complex networks. Rev Mod Phys 74:47-97

Andersen PH, Drejer I, Waldstrøm C (2006) In the eye of the storm-knowledge appropriation and the globalization of regional competencies. In: The proceedings of the annual meeting of the Academy of Management

Chemical Abstracts Service (April 24, 2008) Patent terminology. Available http://www.stn-international.de/training_center/ patents/pat_term.pdf

Chen H, Schuffels C, Orwig R (1996) Internet categorization and search: a machine learning approach. J Vis Commun Image Represent 7(1):88-102

Criscuolo P (2005) The 'home advantage' effect and patent families. A comparison of OECD triadic patents, the USPTO and the EPO. Scientometrics 66(1):23-41

EPO (2008) Global patent data coverage. Available http:// documents.epo.org/projects/babylon/eponet.nsf/0/2464E1 CD907399E0C12572D50031B5DD/\$File/global_patent_ data_coverage.pdf

Espacenet Website. Also published as documents. Retrieved April 24, 2009, from http://ep.espacenet.com/help?topic= publishedas\&amp\&method=handleHelpTopic \&locale $=$ en_ep

Espacenet Website. Corresponding documents. Retrieved April 24, 2009, from http://ep.espacenet.com/help?topic=cor responding\&method=handleHelpTopic\&locale=en_ep
Espacenet Website. Coverage of the worldwide database. Retrieved April 24, 2009, from http://ep.espacenet.com/ help?topic $=$ coverageww

Espacenet Website. esp@cenet databases. Retrieved April 24, 2009, from http://ep.espacenet.com/help?topic=coveragesqh \&locale=en_EP\&method=handleHelpTopic

Espacenet Website. Patent families. Retrieved April 24, 2009, from http://ep.espacenet.com/help?locale=en_EP\&method= handleHelpTopic\&topic=patentfamily

Espacenet Website. What is a cited document? Retrieved April 24, 2009, from http://v3.espacenet.com/answer?AID=3\& AACT $=$ textdoc

European Commission (1997) Second European report on S\&T indicators. European Commission, Brussels

Ganguli P (1998) Intellectual property rights in transition. World Pat Inf 20(3-4):171-180

Garfield E (1955) Citation indexes for science: a new dimension in documentation through association of ideas. Science 122: 108-111

Hingley P, Park W (2003) Patent family data and statistics at the European Patent Office. WIPO-OED workshop on statistics in the patent field, Geneva

Hu D, Chen H, Huang Z, Roco MC (2007) Longitudinal study on patent citations to academic research articles in nanotechnology (1976-2004). J Nanopart Res 9(4):529-542

Huang Z, Chen H, Yip A, Ng G, Guo F, Chen Z-K, Roco MC (2003) Longitudinal patent analysis for nanoscale science and engineering: country, institution and technology field. J Nanopart Res 5:333-363

Huang Z, Chen H, Li X, Roco MC (2006) Connecting NSF funding to patent innovation in nanotechnology (20012004). J Nanopart Res 8(6):859-879

Li X, Lin Y, Chen H, Roco MC (2007) Worldwide nanotechnology development: a comparative study of USPTO, EPO, and JPO patents (1976-2004). J Nanopart Res 9(6):977-1002

Narin F (1998) Patents and publicly funded research: assessing the value of research in the chemical sciences. National Academy Press, Washington, pp 59-72

Oldham P, Cutter AM (2006) Mapping global status and trends in patent activity for biological and genetic material. Genomics Soc Policy 2(2):62-91

Ong T-H, Chen H, Sun W-K, Zhu B (2005) News map: a knowledge map for online news. Decis Support Syst 39(4): 583-597

Oppenheim C (2000) Do patent citations count? The Web of Knowledge (Information Today Inc.), Medford

Roco MC (2005) International perspective on government nanotechnology funding in 2005. J Nanopart Res 7(6):707-712

Roco MC, Williams RS, Alivisatos P (2000) Nanotechnology research directions. Springer, Dordrecht

Seymour EH, Borges FC, Fernandes R (2007) Indicators of European public research in hydrogen and fuel cells-an input-output analysis. Int J Hydrogen Energy 32:3212-3222

Tolle K, Chen H (2000) Comparing noun phrasing techniques for use with medical digital library tools. J Am Soc Inf Sci 51(4):352-370 